\title{
Breast cancer Molecular subtypes and their clinicopathological characteristics amongst patients at the Aga Khan University hospital (Nairobi)
}

Gakinya S.M. MBChB, MMed, Sayed S., MBChB, MMed, Chauhan R., Sayed P. FCPS, PhD, Affiliation: Aga Khan University Hospital, P.O. Box 30270, 00100, Nairobi, Kenya. Correspondence: Dr. Samuel Gakinya

\section{Abstract}

\section{Introduction}

Breast cancer is a heterogeneous disease with varying clinical outcomes in histologically similar tumors. Micro arrays gene profiling has identified several breast cancer subtypes which include luminal A, Luminal B, Basal, and Her2 subtype. These subtypes show variable prognosis and response to therapy.

\section{Objectives}

To determine the proportion of the various subtypes at Aga Khan University hospital and describe their clinical and pathological characteristics.

\section{Study Design \& setting}

Cross sectional study of all breast cancer specimens received in the pathology department of the Aga Khan university Hospital Nairobi between November 2007 and November 2008.

\section{Methods}

101 cases of breast cancer were analysed using immunohistochemical surrogates to identify the subtypes. The subtype definition was; Luminal A ( Estrogen receptor(Er) or Progesterone receptor(Pr) positive
Her2 negative), Luminal B ( Er, Pr, and Her2 positive), Her 2 sub type (Her2 positive, Er and Pr negative), and basal (Er, Pr,and Her2 negative Cytokeratin 5/6 (Ck5/6) and or Her1 positive). Cases not falling into any category were unclassified.

Other clinical pathological characteristics including age, race, menopause status, tumor size, grade and stage were determined in each sub type.

Results

Luminal A was the most common, $42 \%$, followed by basal, Her2, and luminal $B$ at $23 \%, 21 \%$, and $4 \%$ respectively. $10 \%$ of the cases were unclassified. There was no significant difference between the sub types with regard to age, menopause, tumor size and stage. There was a significant difference with regards to grade with the Her2 and basal subtypes having a higher grade.

\section{Conclusion}

The molecular sub types of breast cancer exist in our population. The prevalence of the basal subtype is higher than that seen in studies amongst Caucasians while the prevalence of Luminal $\mathrm{A}$ is lower.

\section{Introduction}

Breast cancer is the second most common malignancy amongst women in Kenya and the most common worldwide. Its incidence in Kenya is estimated to be 1.08 per 100,000 people (1). In 2002, cancer of the breast comprised $20.9 \%$ of new cancers reported at the Nairobi cancer registry (2).

Based on histomorphology, breast cancer is classified into infiltrating ductal carcinoma not other wise specified (NOS), lobular carcinoma, tubular carcinoma, colloid carcinoma, papillary carcinoma, inflammatory carcinoma, micro-papillary carcinoma, cribriform carcinoma, medullary carcinoma, and secretory carcinoma $(3,4)$. Infiltrating ductal carcinoma (NOS) constitutes approximateiy $50 \%$ to $80 \%$ of all breast cancers. This classification is of prognostic importance as some his- tological types such as tubular carcinoma have a better prognosis than invasive ductal carcinoma (NOS) while inflammatory carcinoma has a worse prognosis.

The morphological classification does not however capture the varied clinical heterogeneity of breast cancer as histologically similar tumors classified as ductal carcinoma (NOS) have different clinical course in different patients. This difference may be as a result of molecular variation amongst the cancers such as hormone receptor status and oncogene HER2/neu over expression. Detailed molecular analysis of the cancer would thus be expected to yield additional information that will improve prognostic prediction $(6,7)$.

Several molecular analytical methods have been applied to further sub classify breast cancer. Immunohistochemistry (IHC) was developed 25 years ago and currently 
Breast cancer Molecular subtypes and their clinicopathological characteristics amongst patients at the Aga Khan University hospital (Nairobi) Gakinya S.M., Sayed S., Chauhan R., Sayed P.

forms the cornerstone of molecular classification of breast cancer into ER-positive and ER-negative categories. In addition, over expression of the oncogene HER$2 /$ neu is evaluated using immunohistochemistry and fluorescence in-situ hybridization technique (FISH) and the tumor classified into Her2/neu positive and negative tumors. Hormone receptor positive tumors are responsive to hormonal manipulation and chemotherapy while HER-2/neu positive tumors are responsive to herceptin. $(3,4,5)$.

The development of gene profiling using the microarrays technique has led to the description of distinct molecular subtypes of breast cancer. These subtypes include Luminal A, Luminal B, Basal, and HER2 subtypes. The subtypes are not only different at the molecular level but also at morphological and immunohistochemical level. They also have varying prognoses and response to therapy.

In addition, the molecular signatures that define particular groups may lead to the discovery of new therapeutic targets and treatments that are effective in particular Sub types (6-20).

Our study sought to identify the various molecular subtypes of breast cancer at the Aga Khan University hospital and correlate them with the various clinical and pathological characteristics of the tumor including tumor grade, size, and stage, patient's age, and lymph node status.

\section{Materials and Methods Case selection and analysis of clinical and pathologic characteristics}

A total of one hundred and one well preserved mastectomy specimens were consecutively sampled during the period November 2007 and November 2008.

The tissues were fixed in $10 \%$ formalin, embedded in paraffin blocks then processed for hematoxyllin and eosin (H\&E) staining. Two pathologists reviewed the slides for each case and graded the lesions using the modified Bloom and Richardson classification.

\section{Immunohistochemical techniques}

Immunohistochemical staining was done manually using estrogen receptor (Er) (clone 1D5 Dako), progesterone receptor (Pr) (clone PgR636 Dako), epidermal growth factor receptor 1 (HER1) (Mouse Anti-Her-1 Dako), epidermal growth factor receptor-2 (HER2)
(Polyclonal rabbit Dako), and Cytokeratin 5/6 (Ck5/6) (clone D5/16B4 Dako) primary antibodies.

In brief, 5micron thick sections from representative blocks were deparaffinised in xylene, rehydrated through a series of graded alcohol and rinsed in distilled water. Antigen retrieval using microwave oven heating with $0.1 \mathrm{M}$ citrate buffer was used for all antibodies except Her-1.Enzymatic retrieval using proteinase-K was used for Her-1.

The sections were incubated for 90 minutes with the respective antibodies at the following dilutions; ER (1:50), PR (1:50), HER2 (1:250), HER1 (ready to use), and CK5/6 (1:50). Immunodetection was then done using the Dako envision detection Kit.

The primary antibody incubation step was omitted for the negative control while appropriate control tissues were used as positive controls. In addition, normal breast tissue entrapped within the sections was used as positive control for Er and Pr antibodies.

The slides were counter stained with haematoxyllin.

\section{Interpretation of immunohistochemical staining}

The interpretation of the immunohistochemical staining was reported as negative or positive based on individual antibody staining characteristic. For estrogen and progesterone antibodies, cases were considered positive if at least $10 \%$ of the cells showed nuclei staining of mild to marked intensity. HER2 antibody was considered positive if it showed strong membrane staining in at least $10 \%$ of the cells. HER1 antibody was reported positive if it showed any amount of membrane staining irrespective of number or intensity. Cytokeratin 5/6 antibody was considered positive if any number of cells showed cytoplasm staining irrespective of the intensity.

\section{Definition for molecular subtypes}

The immunohistochemical profile described by Nielsen et.al (21) was applied to define the molecular subtypes. Four subtypes were defined thus: Luminal A (positive for ER or PR and negative for HER2, HER1, and cytokeratin 5/6), Luminal B (positive for ER, PR, and HER2 while negative for HER1 and cytokeratin 5/6), basal subtype (positive for HER1 or Cytokeratin 5/6 and negative for the other markers), and HER2 subtype (positive for HER2 only). Any tumor which did not fit into this category was classified as unclassified. 


\section{Clinical and pathological characteristics}

Clinical and pathologic characteristics evaluated for each case were patient's age, tumor grade, tumor size, pathological tumor stage, and pathological Nodal stage.

The tumor size was defined as the greatest tumor diameter measured grossly.

The pathological tumor stage was determined by assessing the size of tumor and adherence to skin or chest wall muscle while the pathological nodal stage was determined based on the number of nodes with metastasis. These were evaluated by examining the haematoxyllin and eosin stained slides for each sample.

\section{Data Analysis}

Data was analyzed using SPSS version 15 computer software. Proportions were calculated for the various subtypes and also the various clinical pathological characteristics per subtype. The median age and mean tumor size per subtype was determined.

One way analysis of variance (ANOVA) was used to assess for association of age and sub type. A Kruskal-Wallis test was used to assess for association of tumor size and sub types while a Fishers exact test was used for tumor grade, tumor pathological stage and nodal pathological stage.

A P-value of $<0.05$ was considered statistically significant.

\section{Results}

\section{Age}

The median age of the sample was 52 years with the age ranging from 23 to 85 years (Fig. 1). The age group 51 to 55 years had the highest proportion.

\section{Molecular subtypes}

The most common subtype was luminal A with $42 \%$, followed by basal, Her2/neu and luminal B at 23\%, 21\%, and $4 \%$ respectively. Ten percent of the cases did not fit into any of the categories and were thus grouped as unclassified (Fig. 2).

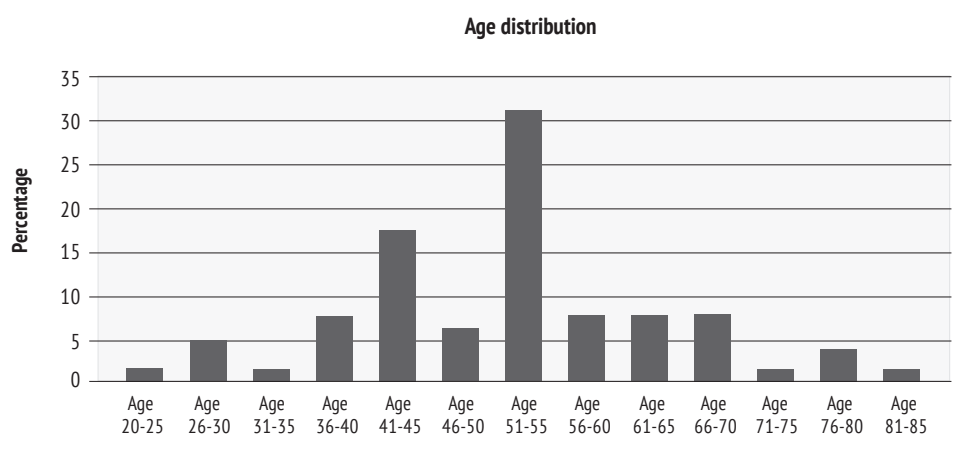

Age

Figure 1: Age distribution

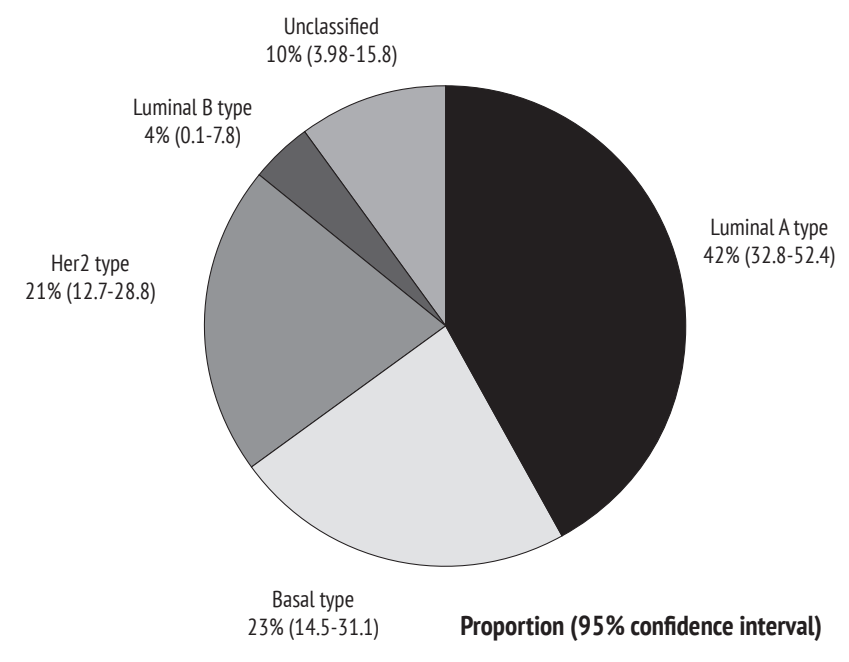

Figure 2: Pie chart showing proportion of subtypes

\section{Breast cancer subtypes and age}

The median age and range in each sub type is shown in the table below. (Table 1).

The median age was not significantly different between the subtypes $(\mathrm{p}=0.944)$.

\section{Breast cancer subtypes and tumor size}

The size of the tumors varied from $1.5 \mathrm{~cm}$ to $20 \mathrm{~cm}$. The mean size for all the tumors was $5.9 \mathrm{~cm}$. Amongst the subtypes, the smallest mean tumor size was $4.7 \mathrm{~cm}$ for the luminal A sub type while the largest was document- 
Breast cancer Molecular subtypes and their clinicopathological characteristics amongst patients at the Aga Khan University hospital (Nairobi) Gakinya S.M., Sayed S., Chauhan R., Sayed P.

\begin{tabular}{lc} 
Sub type & Median age in years (Range) \\
\hline Unclassified & $50(36,77)$ \\
\hline Her2 type & $48(28,71)$ \\
\hline Basal type & $53(30,80)$ \\
\hline Luminal B type & $53(45,57)$ \\
\hline Luminal A type & $49(23,85)$ \\
\hline
\end{tabular}

Table 1: Median age and range per sub type

\begin{tabular}{llc} 
Sub type & Mean size(cm) & 95\% Confidence Interval \\
\hline Luminal A type & 4.7 & $4.0-5.4$ \\
\hline Luminal B type & 5.3 & $3.3-7.2$ \\
\hline Her2 type & 6.5 & $5.0-7.9$ \\
\hline Basal type & 6.2 & $4.9-7.5$ \\
\hline Unclassified & 6.7 & $3.4-9.9$ \\
\hline
\end{tabular}

Table 2: Means of tumor size per sub typ

\begin{tabular}{|c|c|c|c|c|c|c|}
\hline Stage(T) & Luminal A & Luminal B & Her2type & Basal type & Unclassified & Total \\
\hline & $n(\%)$ & $\mathrm{n}(\%)$ & $n(\%)$ & $n(\%)$ & $n(\%)$ & n (\%) \\
\hline $\mathrm{T} 1$ & $4(9.3)$ & 0 & 0 & $2(8.7)$ & 0 & $6(5.9)$ \\
\hline $\mathrm{T} 2$ & $17(39.5)$ & $2(50)$ & $6(28.6)$ & $6(26.1)$ & $5(50)$ & $36(35.6)$ \\
\hline T3 & $12(27.9)$ & $1(25)$ & $7(33.3)$ & $11(47.8)$ & $3(30)$ & $34(33.7)$ \\
\hline T4 & $10(23.3)$ & $1(25)$ & $8(38.1)$ & $4(17.4)$ & $2(20)$ & $25(24.8)$ \\
\hline
\end{tabular}

Table 3: Pathological tumor Stage per sub type

\begin{tabular}{|c|c|c|c|c|c|c|}
\hline Stage(T) & Luminal A & Luminal B & Her2type & Basal type & Unclassified & Total \\
\hline & $n(\%)$ & $n(\%)$ & $\mathrm{n}(\%)$ & $n(\%)$ & $n(\%)$ & $n(\%)$ \\
\hline $\mathrm{T} 1$ & $4(9.3)$ & 0 & 0 & $2(8.7)$ & 0 & $6(5.9)$ \\
\hline $\mathrm{T} 2$ & $17(39.5)$ & $2(50)$ & $6(28.6)$ & $6(26.1)$ & $5(50)$ & $36(35.6)$ \\
\hline T3 & $12(27.9)$ & $1(25)$ & $7(33.3)$ & $11(47.8)$ & $3(30)$ & 34 (33.7) \\
\hline T4 & $10(23.3)$ & $1(25)$ & $8(38.1)$ & $4(17.4)$ & $2(20)$ & $25(24.8)$ \\
\hline
\end{tabular}

Table 4: Grade of tumor per subtype

ed for the Her2 measuring $6.5 \mathrm{~cm}$. (Table 2).

Kruskal Wallis test demonstrated that the differences in tumor sizes between the sub types was not statistically significant $(\mathrm{p}=0.195)$

\section{Breast cancer subtypes and pathological tumor stage}

Only $5.9 \%$ of all the cases were in stage T1. Majority of the cases were stages T2 and T3 (33.6\%and $33.7 \%$ respectively).

$48.8 \%$ of the specimens in Luminal A subtype were stages $\mathrm{T} 1$ and $\mathrm{T} 2$ whereas the basal and HER2 subtypes had $34.8 \%$ and $28.6 \%$ respectively. (Table 3 ).

The tumor stage was not significantly different between the subtypes (fishers exact test $\mathrm{p}=0.755)$.

\section{Breast cancer subtype and tumor grade}

Luminal A had majority (62.8\%) of its tumors in grade 2 while the Her 2 and basal subtypes were mostly grade 3 i.e. $71.4 \%$ and $78.3 \%$ respectively. (Table 4 ).

There was a significant difference in tumor grade between the subtypes (Fishers exact test $\mathrm{p}=0.008$ ). The basal and HER2 sub types were associated with higher tumor grade than luminal A and B.

\section{Breast cancer subtypes and lymph node stage}

Of the 101 breast cancer cases studied, 32 $(31.7 \%)$ did not have lymph nodes submitted. Of the remainder (68.3\%), the number of lymph nodes submitted varied from 1 node to 22 nodes. (Table 5)

The Nodal stage was not significantly different between the subtypes (fishers exact test $\mathrm{p}=0.436$.).

\section{Discussion}

Breast cancer is a heterogeneous disease, encompassing a number of distinct biological entities that are associated with specific morphologic features, immunohistochemical characteristics and clinical behavior.

Micro array gene expression profiling analysis has described at least 5 subtypes of breast cancer which include luminal A, Luminal B, Her-2, normal breast-like and Basal like. These subtypes have been shown to be of prognostic and predictive significance $(6-20,22)$.

This study was done to determine the proportions of the various sub types and describe their clinical and pathological characteristics. The sample population was pre- 
dominantly indigenous Africans from different parts of Kenya. The median age was 52 years.

The most common breast cancer subtype in this study was found to be the hormone receptor positive group, Luminal A (42\%). This is similar to reports from North America and Europe. However, the proportion is higher in Caucasians than what is shown in this study with most studies reporting a higher prevalence $(12,23)$. On the contrary, the proportion of luminal A amongst African Americans

\begin{tabular}{llllll} 
& Luminal A & Luminal B & HER 2 & Basal & Unclassified \\
\hline Nodal status & $\mathrm{N}=30$ & $\mathrm{~N}=4$ & $\mathrm{~N}=13$ & $\mathrm{~N}=14$ & $\mathrm{~N}=8$ \\
\hline $\mathrm{N} 0$ & $13.30 \%$ & & $23.10 \%$ & $33.30 \%$ & $12.50 \%$ \\
\hline $\mathrm{N} 1$ & $40.00 \%$ & $50.00 \%$ & $30.80 \%$ & $20.00 \%$ & $37.50 \%$ \\
\hline N2 & $26.70 \%$ & $50.00 \%$ & $15.40 \%$ & $33.30 \%$ & $37.50 \%$ \\
\hline N3 & $20.00 \%$ & & $30.80 \%$ & $13.30 \%$ & $12.50 \%$ \\
\hline
\end{tabular}

Table 5: Pathological nodal stage per sub type

is lower than the Caucasians while closer to the proportion in this study (12). In their study, Lisa et.al found a prevalence of $47.4 \%$ of luminal A amongst the African Americans.

In local studies evaluating the expression of hormone receptors estrogen and progesterone in breast cancer, the frequency of positive tumors has also been shown to be lower than the frequency amongst Caucasians $(24,25)$. Bird et al found a frequency of 34\% hormone receptor positive tumors. This would correspond to luminal A subtype in the current molecular classification.

The Basal subtype was the second most common subtype (23\%). This proportion is comparable to that seen in studies carried out in other African populations (12, 26). However, in Caucasian populations, the prevalence of the basal subtype tends to be lower $(12,23)$. These findings on luminal and basal subtypes may suggest a racial difference with Africans having a relatively lower prevalence of Luminal A and a higher prevalence of Basal subtype.

The frequency of Her2 subtype was $21 \%$. This is similar to results from other local and international studies (12, 24,25 , and 26). The frequency of the luminal B subtype was the lowest in this study similar to other studies (6, 12).

There was no significant difference between the subtypes with regard to age, pathological tumor and nodal stage and tumor size. This is contrary to what has been described in other studies $(12,22)$. The basal subtype is associated with a lower age while both Her2 and basal sub type are associated with bigger tumor size and tumor pathological stage than the luminal subtypes. The Her 2 sub type is associated with a higher lymph node stage than the other sub types. The lack of significant difference with regard to these characteristics may be due the small sample size.

There was a statistically significant difference between the sub types with regards to tumor grade. Her 2 and Basal subtypes were associated with a higher tumor grade than luminal A and B. This is similar to other studies which have shown that these sub types are associated with aggressive features, including high proliferative capacity (measured by mitotic index), high histological grade and high nuclear grade $(12,22)$. The absence of grade 1 tumors however is noted as these tumors have been described in other studies especially in the Luminal A sub type. Additional studies are necessary to further validate this finding.

\section{References}

1. Bjerregaard B, Kungu A. Breast cancer in Kenya. A histopathologic and epidemiologic study. East Afr Med J 1992. 69:62.

2. G.ZMutuma, A.R Korir. Nairobi cancer registry 2000-2002,Kenya medical research institute. Cancer incidence report. October2006.

3. Rosai J. Rosai and Ackerman's surgical pathology 9th ed. Elselvier Inc., St. Louis, MD,2004.

4. Kumar V, Abbas A, Fausto N. Robbins and Cotran, Pathologic basis of disease. 7th edition W.B Saunders co. 2004.

5. Vincent T. Devita. Cancer principles and practice of oncology. 7th edition, Lippincot Williams and wilkins 2005.

6. Perou CM, Jeffrey SS, van de Rijn M. Distinctive gene expression patterns in human mammary epithelial cells and breast cancers. Proc Natl Acad Sci USA. 1999;96:9:212-7.

7. Sotiriou C, Neo SY, McShane LM. Breast cancer classification and prognosis based on gene expression profiles from a population-based study. Proc Natl Acad Sci USA. 2003;100:10393-8.

8. Van de Rijn M, Perou CM, Tibshirani R. Expression of cy- 
tokeratins 17 and 5 identifies a group of breast carcinomas with poor clinical outcome. Amer J Path 2002;161:1991-6.

9. Banerjee A. Basal-like breast carcinomas: clinical outcome and response to chemotherapy. J of Clin Path 2006;59:729735

10. Mervi Laakso. Basoluminal Carcinoma: A new biologically and prognostically distinct entity between basal and luminal breast cancer clinical cancer research. 2006;12; 4185-4191.

11. Jones C, Ford E, Gillett C. Molecular cytogenetic identification of subgroups of grade III invasive ductal breast carcinomas with different clinical outcomes. Clinical Cancer Research. 2004;10:5988-97.

12. Lisa A. Carey, Perou CM, Livasy CA et al. Race, breast cancer subtypes and survival in the Carolina Breast Cancer Study. JAMA 2006;295:2492-2502.

13. Abd El-Rehim DM, Ball G, Pinder SE, et al. High-throughput protein expression analysis using tissue microarray technology of a large well-characterised series identifies biologically distinct classes of breast cancer confirming recent cDNA expression analyses. Int J of Cancer. 2005; 116:340-50.

14. Lajos Pusztai, Chafika Mazouni. Molecular Classification of Breast Cancer: Limitations and potential The Oncologist. 2006; 11:868-877.

15. Taylor-Papadimitriou, J. Keratin expression in human mammary epithelial cells cultured from normal and malignant tissue: relation to in vivo phenotypes and influence of medium. Journal of Cell Science. 1989; 94:403- 413

16. Abd El-Rehim DM, Pinder SE, Paish CE, et al. Expression of luminal and basal cytokeratins in human breast carcinoma. J of Path 2004; 203:661-71.

17. Sørlie T, Perou CM, Tibshirani R, et al. Gene expression patterns of breast carcinomas distinguish tumor subclasses with clinical implications. Proc Natl Acad Sci USA. 2001; 98:10869-74.
18. Sotiriou C, Wirapati P, Loi S, et al. Gene expression profiling in breast cancer: understanding the molecular basis of histologic grade to improve prognosis. J of the Natl Cancer Institute. 2006;98: 262-272.

19. Perou CM, Sorlie T, Eisen MB, van de Rijn et al. Molecular portraits of human breast tumours. Nature. 2000; 406: $747-$ 752.

20. Sørlie T, Tibshirani R, Parker J, et al. Repeated observation of breast tumor subtypes in independent gene expression data sets. Proc Natl Acad Sci USA 2003;100:8418-23.

21. Nielsen TO, Hsu FD, Jensen K, et al. Immunohistochemical and clinical characterization of the basal-like subtype of invasive breast carcinoma. Clin Cancer Res $2004 ; 10: 5367-74$.

22. Mi-Jung Kim MD, PhD et.al, Clinicopathologic significance of the basal-like subtype of breast cancer: a comparison with hormone receptor and Her2/neu-overexpressing phenotypes. Human pathology. 2006;(37)9.

23. Zarcone M., Rosalba Amodio, et.al. Application of a New classification to a breast tumor series from a populationbased cancer registry. Ann. N.Y. Acad. Sci. 2009; 1155: $222-226$

24. Nyagol J., Nyongo A., Byakika B., Muchiri L. et.al. Routine assessment of hormonal receptor and Her-2 status underscores the need for more therapeutic targets in Kenyan women with Breast cancer. Analytical and quantitative cytology and Histology. 2006;28:2.

25. Bird P.A, Hill A.G, Houssami N. Poor hormone receptor expression in East African Breast cancer: evidence of biologically different disease? Ann of Surg Oncol.2008; 15(7):1983-1988.

26. Nalwoga H, Arnes JB, Wabinga H, Akslen LA. Frequency of the basal-like phenotype in African breast cancer. APMIS. 2007;115:1391-9. 